\title{
Spatial distribution characteristics of human activity intensity in Libo County
}

\author{
Youzhi AN ${ }^{1,2, a}$, Fengtai ZHANG ${ }^{1,2, b}$ and Jiangyu LEI $^{1}$ \\ ${ }^{1}$ School of Geography and Tourism, Guizhou Education University, Guiyang 550018, China \\ ${ }^{2}$ Guizhou Provincial Key Laboratory of Geographic State Monitoring, Guiyang 550018, China \\ aanyouzhi@163.com, ${ }^{b}$ zhfthero@126.com
}

\begin{abstract}
Keywords: Libo County, Human Activity Intensity, Spatial Distribution Characteristics
Abstract: The spatial distribution characteristics of human activity intensity in Libo county are studied in three aspects, including the total distribution characteristics, the distribution characteristics of each township and the spatial autocorrelation analysis based on the calculation of the human activity intensity of the villages and towns in Libo County, which is proposed by $\mathrm{Xu}$ Yong et al. The results showed that the overall human activity index in Libo county was low, and the highest level was only $10.8 \%$. The spatial distribution pattern of human activity intensity is generally characterized by low south and low north, showing a tendency of low, low, medium, high and high distribution from southeast to northwest. The intensity of human activities in the villages and towns has significant global spatial agglomeration (positive correlation) characteristics, the concentration pattern is high (HH) and low (LL), and most of the areas have no obvious agglomeration mode, showing obvious local spatial negative correlation type. The concentrated area of high value is concentrated in the northwest part of the town of Jia Liang and Fang village. The low value agglomeration area is distributed in the southeast of the world natural heritage distribution area and the national natural protection area, mainly concentrated in Dong Tong Township.
\end{abstract}

\section{Introduction}

In the information age of twenty-first Century, the rapid development of science and technology and economy and the development of the relationship between human society and the natural environment have also entered a new historical stage. As one of the important indicators to measure the degree of the human society's effect on the natural environment, more and more scholars have paid more and more attention to and paid attention[1-3].

Human activity intensity refers to the general name for the various development, utilization and protection of human activities to meet their own survival and development[4-5]. From the perspective of the main manifestation of human activity, the perspective of land use, the scholars have deeply discussed the characteristics, driving force and method model of land use change. But when it comes to the study of the degree of land use, it often uses land use rate, land development intensity (the proportion of land area in the land area) and other single index, which leads to the one-sided results, and it is very important to explore integrated index integration research.

Through the analysis of the spatial characteristics of the intensity of human activity in Libo County, this study can guide the relevant research among different regions in Guizhou, and provide a theoretical basis for the sustainable development of ecological health in Libo and Guizhou Province, which is conducive to the protection of the ecological environment in this area, which is in line with the principle of sustainable development.

\section{Research Area}

Libo county is located between the southern part of Guizhou Province, Qiannan Buyi and Miao Autonomous Prefecture. Northeast and Qiandongnan Miao and Dong Autonomous Prefecture of Congjiang County, Rongjiang County, the southeast and the Guangxi Zhuang Autonomous Region's 
Huanjiang County, Nandan County adjacent to the West and Dushan County, the north and the boundary of the three aquarium autonomous counties. The area is 2431.8 square kilometers.

Libo County jurisdiction over 1 streets, 5 towns, 2 townships (of which 1 aquarium townships, 1 Yao township): Yuping street, Jiong Town, Chaoyang Town, Jiliang Town, Maolan Town, Xiaoqi Town, Yao Shan Yao Township, Yao Shan Yao nationality township, the dawn Guan Shui nationality township.

\section{Data and Methods}

This paper intends to calculate the human activity intensity of 17 towns in Libo County by using the method of calculating the human activity intensity proposed by $\mathrm{Xu}$ Yong and others - the percentage of land area in the total area of land in the area of Libo County, and subdivides the 17 villages and towns into four different levels of human activity intensity by the natural fracture method, and then further interprets the people of Libo County by the spatial autocorrelation analysis method. The overall distribution and characteristics of the intensity of the activity of the class. Then we analyze the spatial distribution characteristics of different regions.

\section{The calculation method of human activity intensity}

It is based on the change of natural cover under different land use types and the normal exchange of air, heat, moisture and nutrients, forming two levels of 8 characteristic signs and corresponding eigenvalues, and taking this as the calculation standard. The final determination of the equivalent conversion coefficient of the construction land equivalent is shown in the literature. The formula for calculating the intensity of human activity is as follows:

$$
\begin{gathered}
H A I_{i}=\frac{S_{C L E-i}}{S_{i}} \times 100 \% \\
S_{C L E-i j}=\sum_{j=1}^{m}\left(S L_{i j} \times C I_{i j}\right)
\end{gathered}
$$

$H A I i$ is the intensity of human activity in the $i$ area; SCLE- $i$ is the equivalent area of construction land for the I area; $S i$ is the total area of land in the I region; SLij is the area of the third category of the land use type in the I region; CIij is the equivalent conversion factor of the construction land use type of the third region of the $i$ region.

\section{Spatial Autocorrelation Analysis}

Using the Local Moran's I (LISA) method, the local spatial autocorrelation analysis is carried out through the LISA cluster diagram. The LISA value of the LISA cluster diagram must be tested for the $\mathrm{Z}$ value, including the four spatial distribution patterns of high (HH), high and low (HL), low (LH) and low (LL)). These four modes represent the different correlation between each unit and the surrounding unit, such as high type representation.

\section{Sources of Data}

The data of this study are mainly land use vector data and Libo County HD image data, as well as Libo County DEM raster data. The selected remote sensing data is mainly Googel Earth HD image data with spatial resolution of 0.27 meters. According to all data, we can choose the latest time data available, and select the final time of 2013 image data. The data of land use in Libo county is obtained by manual tailoring and digitalization. 


\section{Results}

\section{Integral Distribution Characteristics}

Based on the Arc GIS internatural breakpoint classification method (Jenks), the classification standard of human activity intensity in different towns in Libo county is determined - the unified grading standard is $2.5 \%-3.1 \%, 3.2 \%-4.4 \%, 4.5 \%-6.6 \%, 6.7 \%-8.6 \%$ and $8.7 \% 10.8 \%$, and divides the activity intensity of each Township in Libo County into low, low, medium, high, and higher grade. The overall human activity index in Libo county is low, and the highest level is only $10.8 \%$. This data shows that the overall ecological environment of Libo county is relatively good.

From the overall distribution pattern (Fig.1), the spatial distribution pattern of human activity intensity in Libo county is generally characterized by low south and low north, and the human activities of Jiarong town in the northeast corner are also characterized by low intensity. The intensity distribution of human activities showed a trend of low, low, medium, high and high distribution from southeast to northwest. The lowest value of the forest accounts for more than $80 \%$ of the forests and the towns and villages with low urban and rural construction land, that is, Jiong town in the northeast corner and Weng onong Township and Dong Tong Township in the southeast.

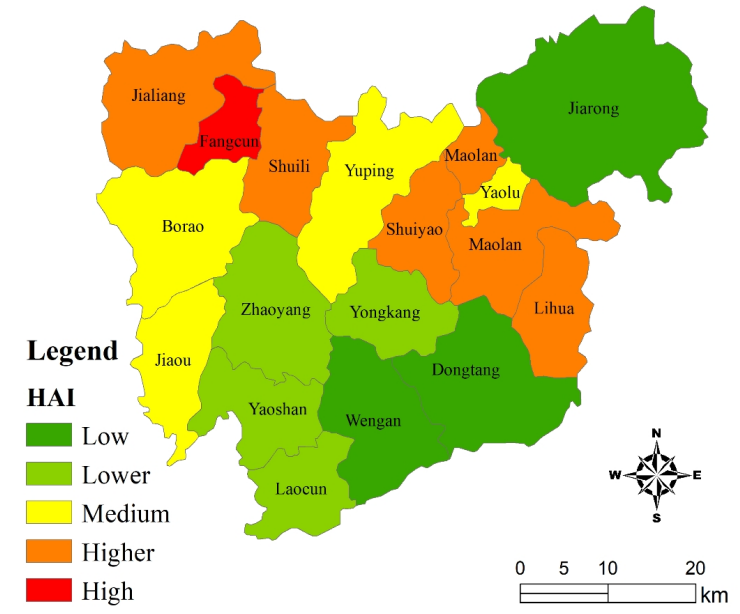

Fig.1 Libo county is divided into different figure of human activity intensity space

\section{Distribution Characteristics of Villages and Towns}

From the view of Figure 1, the highest intensity of human activity is square village, followed by the high level of Fang village, water conservancy and Shui nationality township, Shui Yao and Shui nationality township, Maolan town and Lihua town. The intensity of human activities in Yuping Town, Yao Lu Yao Township, driving Europe Township and Yao Yao township is at the middle level; the intensity of human activities in Chaoyang, Yao nationality township, Yao Shan Yao Township, Yao Shan Yao Township and Village Township is low level; human activities are strong. Is the lowest, Angxiang, Weng Jia Rong Zhen Dong Tang xiang. From the point of view of this distribution, the low level and lower level of human activity in Libo county are mainly distributed in the towns under the jurisdiction of the world natural heritage area and the National Nature Reserve, as well as the large area of Jiarong Town, while the high level of human activity and the higher type are mainly distributed in the relatively low proportion of the forest area in the township and the relatively high proportion of the cultivated land area. Libo county is located in Yuping County, where human activities are in medium grade intensity. 


\section{Space Autocorrelation Analysis}

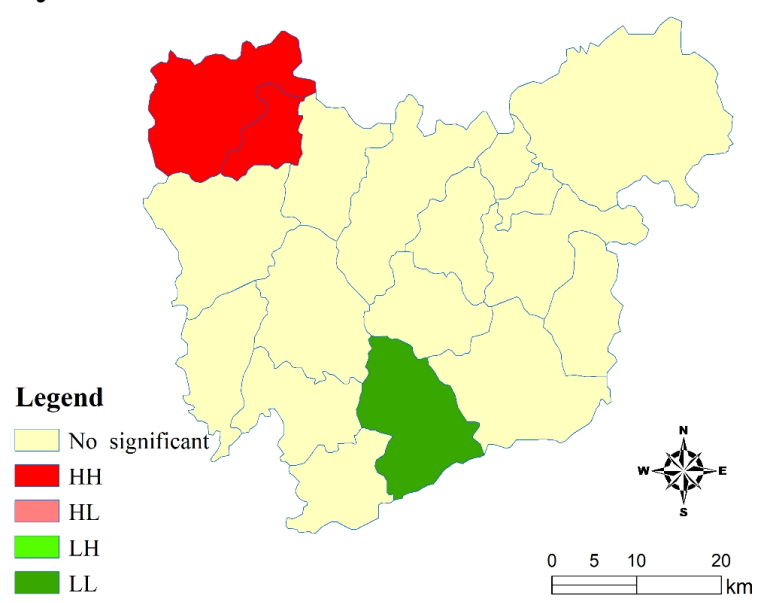

Fig. 2 LISA concentration map of human activity intensity in Libo County

Figure 2 is the LISA concentration map of the human activity intensity in Libo county (through $\mathrm{Z}$ value test, $\mathrm{P}<0.01$ ). According to this chart, it can be seen that the clustering and abnormity analysis of the spatial autocorrelation analysis of human activity intensity in Libo counties show that the model is high (HH) and low (LL), and there is no obvious agglomeration mode in most areas, showing obvious local spatial negative correlation types. In addition, according to the high value area of high type of human activity, the intensity of human activity in Libo county shows relatively high distribution characteristics around the town of Jia Liang and Fang village, and there is a significant positive correlation between the local space and the local space. According to the low value area of low and low type of human activity, the intensity of human activity in Libo county is also relatively low around Weng Aung Township, and there is also a positive correlation between the high and high type areas.

\section{Conclusions}

(1) The overall human activity index in Libo county is relatively low, and the highest level is only $10.8 \%$. The spatial distribution pattern of human activity intensity generally shows the characteristics of South low and north high.

(2) From the distribution characteristics of the villages and towns, the low level and lower level of human activities in Libo county are mainly distributed in the towns under the jurisdiction of the world natural heritage area and the National Nature Reserve, as well as the vast area of Jiarong Town, while the high level of human activity intensity and the higher types are relatively low in the proportion of the forest area and the proportion of the cultivated land area.

(3) According to the results of spatial autocorrelation analysis, the intensity of human activities in towns and townships in Libo has a significant global spatial concentration (positive correlation) characteristics. The concentration pattern of human activity intensity in each township is mainly high $(\mathrm{HH})$ and low (LL), and there is no obvious agglomeration mode in most of the areas, and there are obvious negative correlation types of local space.

\section{Acknowledgements}

This work was financially supported by the Science and Technology Fund of Guizhou Province, China (grant no. [2017]1135); the Science and Technology Fund of Guizhou Province, China (grant no. [2015]2121), and the Science and Technology Fund of Guizhou Province, China (grant no. [2016]ZHCH2845). 


\section{References}

[1] JIANG Dong , YAN Xiaoxi , FU Jingying. Applicability comparison of human activity extraction by multi-scale remote sensing imageries : A case study in Lingwu Baijitan National Nature Reserve. Resources Science Vol. 38(2016), p. 1409-1422

[2] WU Linna, YANG Shengtian, LIU Xiaoyan, LUO Ya, ZHOU Xu, ZHAO Haigen. Response analysis of land use change to the degree of human activities in Beiluo River basin since 1976. Acta Geographica Sinica Vol. 69(2014), p. 54-63

[3] HUANG Yaohuan , ZHAO Chuanpeng , YANG Haijun , DING Fangyu , LI Zhonghua. Spatial distribution and aggregation analysis of human activity in national key ecological function regions in China Resources Science Vol. 38 (2016), p. 1423-1433

[4] XU Xiaoren, XU Yong. Analysis of spatial-temporal variation of human activity intensity in Loess Plateau region. Geographical Research Vol. 36 (2017), p. 661-672

[5] YE Chun, LI Chunhua, WU Lei, YANG Wenjuan. Ecological Degradation of Lake Littoral Zone and Interaction Effects with Human Activities. Research of Environmental Sciences Vol. 28 (2015), p. 401-407 